\title{
Cochlear Sparing for Brain Tumor Radiotherapy: A Retrospective Study Comparing Intensity-Modulated Radiotherapy and Volumetric Modulated Arc Therapy
}

\author{
(1) Gülşen Pınar SOYDEMIR GÖÇER, (1) Elif Eda ÖZER
}

Department of Radiation Oncology, Bakırköy Dr. Sadi Konuk Education and Research Hospital, İstanbul-Turkey

\begin{abstract}
OBJECTIVE
Radiation-induced hearing loss is a common complication of radiotherapy in patients with brain tumors. This retrospective study aims to compare Intensity-Modulated Radiotherapy (IMRT) with Volumetric Modulated Arc Therapy (VMAT) for the patients with brain tumor concerning the sparing effect on the cochlea by comparing dosimetric parameters.
\end{abstract}

\section{METHODS}

In this study, 20 patients with pathological grade IV brain tumors were selected to have planning with VMAT and IMRT for dosimetric comparison concerning organs at risk. Target coverage and dose homogeneity were evaluated by calculating the conformity index (CI) and homogeneity index (HI) values. The treatment plans were accepted only if they met the set of planning objectives defined in the protocol.

\section{RESULTS}

The homogeneity and conformity of the two plans were statistically similar. The dose to receive $95 \%$ $\left(\mathrm{D}_{95 \%}\right)$ of planning target volume (PTV) and the minimum dose received $46 \mathrm{~Gy}\left(\mathrm{D}_{\min }\right)$ were significantly lower with IMRT compared with VMAT ( $\mathrm{p}=0.01$ and 0.001 , respectively), but the volume receiving 46 $\mathrm{Gy}\left(\mathrm{V}_{46 \mathrm{~Gy}}\right)$ and $60 \mathrm{~Gy}\left(\mathrm{~V}_{60 \mathrm{~Gy}}\right)$, and $\mathrm{D}_{95 \%}, \mathrm{D}_{\text {mean }}, \mathrm{D}_{\max }, \mathrm{D}_{\min }$ received $60 \mathrm{~Gy}$ were not different between two methods. There was no significant difference in $\mathrm{D}_{95 \%}, \mathrm{D}_{\text {mean }}$, and $\mathrm{D}_{\max }$ for total and contralateral cochleae among groups.

\section{CONCLUSION}

Since VMAT and IMRT planning have the same effect on sparing the cochlea; no superiority can be suggested among these two plans during radiotherapy. The choice of plan for the treatment of brain tumors appears to base on the clinical experience, especially for patients who may experience hearing loss.

Keywords: Brain tumor; cochlea; IMRT; radiotherapy; VMAT.

Copyright $\odot$ 2020, Turkish Society for Radiation Oncology

\section{Introduction}

Approximately $10 \%-30 \%$ of the cancer patients have brain metastases, which are the most common intracranial tumors.[1] The standard treatment choice in brain tumors is the maximal surgical resection, followed by adjuvant radiotherapy. Combining radiotherapy with chemotherapy (concomitant and adjuvant temozolomide) has revealed a higher survival benefit and lower additional toxicity in patients with high-grade brain tu- 
mors. However, radiotherapy may have side effects on the brain, including radiation necrosis, cognitive impairment, and a high chance of tumor recurrence, which has been considered the most common cause of treatment failure.[2] Moreover, there is evidence about the radiation therapy for tumors in the head and neck region, showing a correlation between the dose to the cochlea and the degree of hearing loss observed.[3-5] At dose levels of radiotherapy applied to most of the intracranial brain tumors, the cochlea is the main organ affected and the mean dose to the cochlea $\left(\mathrm{D}_{\text {mean }}\right)$ became an essential factor mostly studies to determine the incidence and degree of hearing loss.[3,5] Thus, different strategies of dose prescription and modern planning techniques have been tested to achieve better tumor control and better sparing of the organs at risk (OARs).[6,7] The most common strategies for RT are intensity-modulated radiation therapy (IMRT) and volumetric-modulated arc therapy (VMAT). VMAT is a subset of IMRT where radiation is delivered in an arc instead of at static angles, allowing the delivery of highly conformal dose distributions. The idea of these techniques has arisen to selectively spare the cochlear structures and to reduce the risk of radiation-induced complications such as hearing loss during brain radiotherapy. [8]

IMRT technique delivers variable intensity radiation with multiple radiation beams; thus, target volume conformity and sparing of normal tissues and OARs have been improved considerably.[9] Moreover, IMRT is able to produce inhomogeneous dose distributions, resulting in the simultaneous delivery of different doses per fraction to separate areas within the target volume. On the other hand, VMAT allows simultaneous variation of three parameters during the delivery of radiation therapy, i.e., gantry rotation speed, treatment aperture shape via movement of multileaf collimator (MLC) leaves, and dose rate. This causes further improvement in target volume conformity and OARs sparing.[10] In this study, we aimed to test the potential outcomes of sparing the cochlea in radiotherapy to brain tumors in 20 patients using VMAT and IMRT techniques and set various dosimetric objectives to compare dosimetric parameters between VMAT and IMRT.

\section{Materials and Methods}

\section{Selection and Description of the Patients}

In total, 20 patients diagnosed with pathological grade IV brain tumor were retrospectively enrolled in this retrospective comparative clinical study. The mean age of the patients was $52.1 \pm 8.64$ years (range, 34-61 years). All patients underwent preoperative MRI or CT. The present study was conducted in accordance with the Ethics Committee Approval (Number: 2017/28) for VMAT/IMRT planning and with the principles of the WMA Declaration of Helsinki. Patient consent was not obligatory, as stated by the Ethics Committee of our institute.

Datasets of 20 patients who had received either IMRT or VMAT for a brain tumor at our institute were included in this planning study. For all patients, the dose prescribed to the planning target volume (PTV) was 46 Gy followed by a boost of 60 Gy in 30 fractions. Patients were positioned in the supine position and immobilized with a thermoplastic mask. Normal structures like the eyes, lenses, optic nerves, chiasm, cochlea and brain stem were contoured and designated as OARs (Fig. 1). Expanded contours were created with safety margins of $3 \mathrm{~mm}$ around the brain stem and chiasm and $5 \mathrm{~mm}$ around the optic nerve. The cochlea was contoured on the T1 MRI sequence, as described by Gao et al.[11]

\section{VMAT Planning}

The VMAT plans were optimized in the research treatment planning system (TPS) of Monaco ${ }^{\circledR} 5.1$ (Elekta AB Publ, Stockholm, Sweden), which relies on the XVMC (X-ray voxel Monte Carlo) algorithm for dose calculation.[12] All VMAT plans were generated for a $6 \mathrm{MV}$ Elekta Synergy linear accelerator (LINAC) equipped with an Agility MLC. Three fields were used with a gantry angle beginning from $180^{\circ}$. The first field was given with both clockwise and counter clockwise gantry angle of $160^{\circ}$ on the basis of tumor location, and as the eyeballs and lenses of the patients did not

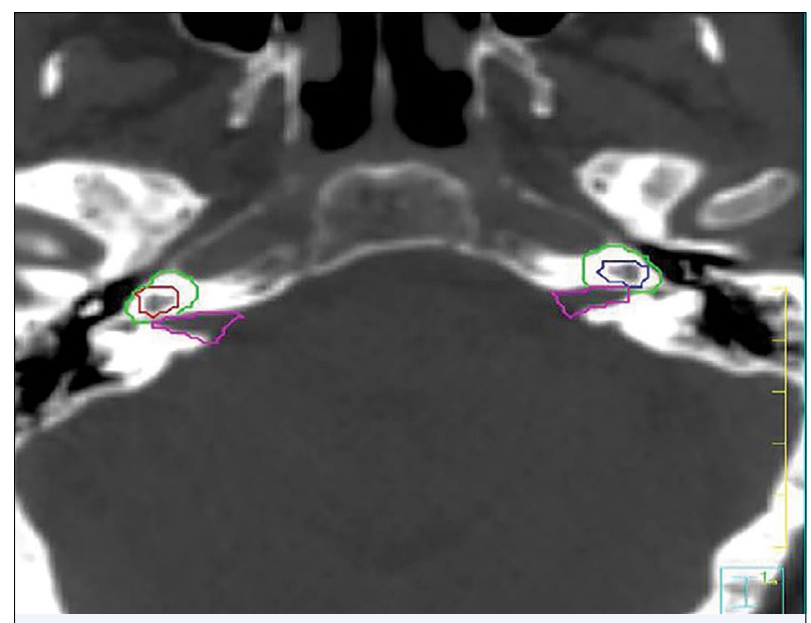

Fig. 1. A radiographic image of the cochlea. 
take the initial dose. Moreover, the collimator angles were given in between $50^{\circ}$ and $70^{\circ}$ on the basis of tumor location. The second field was given in between $40^{\circ}$ and $50^{\circ}$ as all the PTVs were caught, the lens and eyes were excluded from the exit dose, and the collimator angle was given in between $50^{\circ}$ and $70^{\circ}$. The third field was given as half $\operatorname{arc}\left(180^{\circ}\right)$ with a table angle of $55^{\circ}$ and $305^{\circ}$ on the basis of tumor location to protect the healthy tissues of the patients.

For Monte Carlo-based VMAT planning for the patients, two arcs for each field, 180 control points for each arc, statistic as one, and grid size as 0.3 were chosen.

\section{IMRT Planning}

For generating IMRT plans for the patients, ELECTA TPS of Elekta Monaco 5.1, which relies on the XVMC algorithm (X-ray voxel Monte Carlo), was used for dose calculation. All IMRT plans were generated in a 6 MV Elekta Synergy LINAC, equipped with an Agility MLC. IMRT planning was performed with six fields using a multileaf collimator delivery method in dynamic mode. The initial gantry angles of these six fields were chosen as $260^{\circ}, 230^{\circ}, 170^{\circ}, 140^{\circ}, 110^{\circ}$, and $70^{\circ}$. Collimator or table angle was not used for any of these fields.

\section{Evaluation of Treatment Plans}

Monte Carlo algorithm was used for both plans. After contour radiation was entered by the oncologist, VMAT and IMRT plans were performed by a medical physicist. VMAT plans were performed twice to spare and not to spare (standard) the cochlea contour in optimized condition. For both VMAT plans, the same angles and cost functions were used. The maximum control point for each arc used in the VMAT plan was chosen as 180 .

The IMRT plans were performed twice to spare and not to spare (standard) the cochlea contour in optimized condition. For both IMRT plans, the same angles and cost functions were used. The planning of these IMRT plans was adjusted at 15 segments of each field.

For both techniques, cochlear sparing was targeted as PTV covering $100 \%$ of the volume at $95 \%$ of the target volume dose. In plans where $95 \%$ of PTV was covered by $100 \%$ of target volume dose, the conformity index enhancer, planned template additional field, or cost functions were not used.

\section{Statistical Analysis}

All dosimetric values were reported in descriptive statistics as mean $\pm \mathrm{SD}$ and median (minimum-maxi-

mum) values. Dosimetric parameters were tested for normal distribution using the Kolmogorov-Smirnov test. Because two different plans were generated in the CT image set of every patient, the data were considered matched pair and paired tests were used to compare the two plans. For two non-normally distributed dependent variables, a corresponding non-parametric test Wilcoxon test was used, and for more than two non-normally distributed dependent variables, the Friedman test was used. All statistical analyses were performed with a $5 \%$ level of significance, and $p<0.05$ was considered statistically significant. The analysis was performed using the MedCalc Statistical Software version 12.7.7 program (MedCalc Software bvba, Ostend, Belgium; http://www.medcalc.org; 2013).

\section{Results}

The VMAT and IMRT plans met the planning objectives for 20 patients ( 10 male and 10 female), as mentioned in the protocol. The characteristics and total dosimetric results of the patients are given in Table 1. The mean age of the patients who underwent total $(n=18)$ or subtotal $(n=2)$ brain surgery was $52.1 \pm 8.64$ years, and the tumors were located in the temporal $(n=6)$, parietal $(n=8)$, frontal $(n=4)$ and parieto-occipital $(n=2)$ regions of the brain. The median clinical target (CT) volume was $161.34 \mathrm{~cm}^{3}$ (min-max: 41.95$320)$.

Table 1 Demographics of the patients $(n=20)$

\section{Characteristics}

\begin{tabular}{lc}
\hline Age (year) & \\
Mean \pm SD & $52.1 \pm 8.64$ \\
Median (Min-Max) & $54.5(34-61)$ \\
Gender & $\mathrm{n}(\%)$ \\
Female & $10(50)$ \\
Male & $10(50)$ \\
Operation type & $\mathrm{n}(\%)$ \\
Total & $18(90)$ \\
Subtotal & $2(10)$ \\
Localization of brain tumor & $\mathrm{n}(\%)$ \\
Temporal & $6(30)$ \\
Parietal & $8(40)$ \\
Frontal & $4(20)$ \\
Parieto-occipital & $2(10)$ \\
CT volume & \\
Mean \pm SD & $172.47 \pm 105.46$ \\
Median (Min-Max) & $161.34(41.95-320)$ \\
\hline
\end{tabular}

CT: Clinical target, SD: Standard devision 
The dosimetric data obtained from generated dosevolume histograms are presented in Table 2 . When the dosimetric parameters of PTV were compared at the coverage of the treatment target dose at $46 \mathrm{~Gy}$, the

\begin{tabular}{|c|c|c|c|}
\hline ble 2 & $\begin{array}{l}\text { Dosimetric comp } \\
\text { IMRT }(n=10) \text { plar } \\
\text { volumes }\end{array}$ & $\begin{array}{l}\text { son of the VMAT ( } \mathrm{n} \\
\text { r different plannin }\end{array}$ & $\begin{array}{l}\text { ) and } \\
\text { rget }\end{array}$ \\
\hline Variable & $\begin{array}{c}\text { VMAT } \\
\text { Mean } \pm \text { SD } \\
\text { Median (Min-Max) }\end{array}$ & $\begin{array}{c}\text { IMRT } \\
\text { Mean } \pm \text { SD } \\
\text { Median (Min-Max) }\end{array}$ & $\mathbf{p}$ \\
\hline $\mathrm{V}_{46 \mathrm{~Gy}}$ & $\begin{array}{r}\mathrm{PT} \\
412 . \\
394.91(34\end{array}$ & $\begin{array}{l}46 G y \\
7 \pm 4.03 \\
.45-593.22)\end{array}$ & 1.000 \\
\hline$D_{95 \%}$ & $\begin{array}{c}4615.5 \pm 28.11 \\
4614.5(4565-4652)\end{array}$ & $\begin{array}{c}4570.2 \pm 36.24 \\
4585(4500-4600)\end{array}$ & $0.010^{*}$ \\
\hline$D_{\text {mean }}$ & $\begin{array}{c}4691.2 \pm 25.6 \\
4692.5(4662-4722)\end{array}$ & $\begin{array}{c}4701.3 \pm 38.95 \\
4714(4641-4761)\end{array}$ & 0.406 \\
\hline $\mathrm{D}_{\max }$ & $\begin{array}{c}4995.2 \pm 33.13 \\
5007(4928-5025)\end{array}$ & $\begin{array}{c}5019.5 \pm 47.21 \\
5021(4937-5080)\end{array}$ & 0.199 \\
\hline$D_{\min }$ & $\begin{array}{c}4114.9 \pm 240.79 \\
4165(3586-4400)\end{array}$ & $\begin{array}{c}3295 \pm 467.29 \\
3400.5(2502-3997)\end{array}$ & $0.001 *$ \\
\hline$V_{60 G y}$ & $\begin{array}{r}\mathrm{P} \\
294 \\
275.58(18\end{array}$ & $\begin{array}{l}{ }_{60 G y} \\
9 \pm 81.2 \\
.16-431.38)\end{array}$ & 1.000 \\
\hline$D_{95 \%}$ & $\begin{array}{c}5940.8 \pm 106.88 \\
5975.5(5710-6061)\end{array}$ & $\begin{array}{c}5945.9 \pm 74.17 \\
5922(5830-6079)\end{array}$ & 0.850 \\
\hline$D_{\text {mean }}$ & $\begin{array}{c}6085.9 \pm 45.07 \\
6087(5994-6158)\end{array}$ & $\begin{array}{c}6142.7 \pm 72.43 \\
6144(6011-6246)\end{array}$ & 0.059 \\
\hline $\mathrm{D}_{\max }$ & $\begin{array}{c}6405.1 \pm 43.23 \\
6407.5(6345-6461)\end{array}$ & $\begin{array}{c}6439.2 \pm 93.84 \\
6440(6284-6550)\end{array}$ & 0.326 \\
\hline$D_{\text {min }}$ & $\begin{array}{c}5011.4 \pm 431.6 \\
4967(4389-5659)\end{array}$ & $\begin{array}{c}4755.7 \pm 426.17 \\
4697.5(4215-5504)\end{array}$ & 0.241 \\
\hline & & & \\
\hline$D_{98 \%}$ & $\begin{array}{c}5804.5 \pm 195.39 \\
5834.5(5535-6025)\end{array}$ & $\begin{array}{c}5801.5 \pm 150.4 \\
5809.5(5611-6018)\end{array}$ & 0.940 \\
\hline$D_{95 \%}$ & $\begin{array}{c}5939.9 \pm 106.09 \\
5975.5(5710-6061)\end{array}$ & $\begin{array}{c}5945.9 \pm 74.17 \\
5922(5830-6079)\end{array}$ & 0.880 \\
\hline$D_{5 \%}$ & $\begin{array}{c}6205.8 \pm 36.07 \\
6212(6138-6259)\end{array}$ & $\begin{array}{c}6255.8 \pm 98.02 \\
6273.5(6096-6400)\end{array}$ & 0.162 \\
\hline$D_{2 \%}$ & $\begin{array}{c}6233.6 \pm 35.18 \\
6238(6164-6282)\end{array}$ & $\begin{array}{c}6282.6 \pm 99.26 \\
6302(6120-6432)\end{array}$ & 0.140 \\
\hline $\mathrm{Cl}$ & $\begin{array}{c}0.81 \pm 0.07 \\
0.81(0.71-0.91)\end{array}$ & $\begin{array}{c}0.8 \pm 0.05 \\
0.8(0.73-0.89)\end{array}$ & 0.970 \\
\hline $\mathrm{HI}$ & $\begin{array}{c}1.05 \pm 0.02 \\
1.05(1.02-1.08)\end{array}$ & $\begin{array}{c}1.05 \pm 0.02 \\
1.05(1.02-1.09)\end{array}$ & 0.673 \\
\hline MU & $\begin{array}{c}639.05 \pm 77.26 \\
630.7(550.3-773.5)\end{array}$ & $\begin{array}{c}546.52 \pm 84.46 \\
567.55(368.3-671.2)\end{array}$ & $0.034^{*}$ \\
\hline
\end{tabular}

*P $<0.05$, compared with Mann Whitney $\mathrm{U}$ test, VMAT: Volumetric Modulated Arc Therapy, IMRT: Intensity-Modulated Radiotherapy, PTV: Planning target volume, $\mathrm{D}_{95 \%}$ : The dose to receive $95 \%$ of PTV ${ }_{46 G \mathrm{y}}$ or $60 \mathrm{~Gy}, \mathrm{D}_{\text {min }}$ : Minimum dose, $\mathrm{D}_{\text {mean }}$ mean dose, $\mathrm{D}_{\max }:$ Maximum dose, $\mathrm{V}_{\mathrm{XG}}:$ Volume receiving $\mathrm{X} G \mathrm{G}$ or more, $\mathrm{D}_{x}^{\text {mean }}$ The dose to receive $\mathrm{X} \%$ of PTV, Cl: Conformity index, HI: Homogeneity index, MU: Monitor unit whole target receiving at least $95 \%$ of the prescription dose $\left(\mathrm{D}_{95 \%}\right)$ was statistically higher in VMAT plans compared to the IMRT plans $(\mathrm{p}=0.010)$. The minimum dose at PTV 46 Gy was also significantly higher in the VMAT plan than the IMRT plan ( $\mathrm{p}=0.001)$, while there was no statistical difference in mean and maximum dose at PTV 46 Gy among two treatment modalities. However, the dose to receive at least $95 \%$ of $60 \mathrm{~Gy}$ and the mean, maximum and minimum doses at PTV 60 Gy were comparable between VMAT and IMRT plans (Table 2). The doses to receive 98\%, 95\%, 5\% and 2\% of PTV were also comparable between the two methods. The conformity and homogeneity indexes of two methods were the same; however, a significantly higher monitor unit (MU) was noted in the VMAT plan when compared with the IMRT plan $(\mathrm{p}=0.034)$.

All VMAT and IMRT plans were able to meet the constraints placed on OARs and PTV. When dosimetric variables for OARs were compared, no significant difference was found for the dose to cover $95 \%$ of both contralateral and total cochlea among VMAT and IMRT plans, even the mean and maximum doses to cochlear structures did not differ among two methods.

Considering PTV 46 Gy (Table 3), the doses to the left eye and left optic nerve were found to be higher in the IMRT plan ( $\mathrm{p}=0.041$ and 0.023 , respectively), while the doses to the optic chiasm and brain stem were higher in the VMAT plan ( $\mathrm{p}=0.001$ and 0.002 , respectively). The same difference was also seen in the doses to the left eye and left optic nerve at PTV 60 Gy that is to note considerably higher doses in the IMRT plan compared to the VMAT plan ( $\mathrm{p}=0.019$ for both). However, the doses to the optic chiasm and brain stem were nearly similar between two modalities. Other doses to the right eye, both lenses and right optic nerve, were statistically comparable for both plans.

\section{Discussion}

Brain tumors are one of the aggressive tumors carrying poor prognosis in cancer patients. An estimated 15\% to $30 \%$ of these cancer patients will develop brain metastases during the course of their illness.[13] A multimodality treatment approach, including surgical intervention, radiotherapy and/or stereotactic radiosurgery (SRS) for intracranial tumors, may result in improvement in local control and neurologic symptoms of the disease and expectantly in quality of life and survival of patients.[14] With advanced radiation technology, such as VMAT and IMRT techniques, it is possible to carry out radiotherapy with cochlear sparing and re- 
Table 3 Dosimetric comparison of the VMAT $(n=10)$ and IMRT $(n=10)$ plans for organs at risk

Variable
Mean \pm SD
Median (Min-Max)

\section{IMRT \\ Mean \pm SD \\ Median (Min-Max)}

\begin{tabular}{|c|c|c|c|}
\hline \multirow{3}{*}{$D_{95 \%}$} & \multicolumn{2}{|c|}{ Contralateral cochlea } & \multirow[b]{3}{*}{0.545} \\
\hline & $8.54 \pm 5.03$ & $11.6 \pm 9.75$ & \\
\hline & $9.62(1.83-15.81)$ & $11.24(1.82-29.58)$ & \\
\hline \multirow{2}{*}{$D_{\text {mean }}$} & $10.04 \pm 5.5$ & $13.86 \pm 10.37$ & \\
\hline & $11.78(2.05-16.88)$ & $14.19(2.06-31.22)$ & 0.406 \\
\hline \multirow{3}{*}{$\mathrm{D}_{\max }$} & $12.2 \pm 6.25$ & $16.5 \pm 11.36$ & \\
\hline & $14.84(2.34-19.3)$ & $17.14(2.45-33.13)$ & 0.364 \\
\hline & & & \\
\hline \multirow{2}{*}{$D_{95 \%}$} & $8.7 \pm 5.1$ & $11.63 \pm 9.5$ & \\
\hline & $9.84(1.89-15.91)$ & $11.43(1.84-29.78)$ & 0.450 \\
\hline \multirow{2}{*}{$D_{\text {mean }}$} & $19.52 \pm 13.17$ & $20.71 \pm 14.77$ & \\
\hline & $21.27(2.4-36.21)$ & $21.34(2.52-42.42)$ & 0.762 \\
\hline \multirow{2}{*}{$\mathrm{D}_{\max }$} & $35.26 \pm 22.88$ & $34.57 \pm 22.27$ & \\
\hline & $44.98(3.4-61.19)$ & $41.48(3.61-62.59)$ & 0.940 \\
\hline \multirow{3}{*}{ Right eye } & & & \\
\hline & $21.44 \pm 9.09$ & $17.39 \pm 5.9$ & \\
\hline & $20.31(8.33-36.46)$ & $15.91(8.95-28)$ & 0.326 \\
\hline \multirow[t]{2}{*}{ Left eye } & $15.92 \pm 8.13$ & $25.06 \pm 6.12$ & \\
\hline & $12.75(6.86-33.82)$ & $24.79(10.5-33.52)$ & $0.041^{*}$ \\
\hline \multirow[t]{2}{*}{ Right lens } & $5.31 \pm 0.45$ & $5.35 \pm 0.44$ & \\
\hline & $5.44(4.27-5.8)$ & $5.54(4.53-5.78)$ & 0.597 \\
\hline \multirow[t]{2}{*}{ Left lens } & $5.29 \pm 0.81$ & $5.61 \pm 0.53$ & \\
\hline & $5.52(3.18-5.96)$ & $5.6(4.97-6.68)$ & 0.762 \\
\hline \multirow[t]{2}{*}{ Right optic nerve } & $30.06 \pm 10.03$ & $25.2 \pm 7.64$ & \\
\hline & $34.64-(14.44-41.51)$ & $25.61(13.27-38.46)$ & 0.290 \\
\hline \multirow[t]{3}{*}{ Left optic nerve } & $(n=10)$ & $(n=10)$ & \\
\hline & $23.85 \pm 9.01$ & $29.79 \pm 5.96$ & \\
\hline & $20.18(15.77-41.13)$ & $29.7(23.63-42.74)$ & $0.023^{*}$ \\
\hline \multirow[t]{2}{*}{ Optic chiasm } & $43.79 \pm 1.52$ & $41.05 \pm 1.13$ & \\
\hline & $43.6(40.69-46.14)$ & $40.68(39.53-42.94)$ & $0.001^{*}$ \\
\hline \multirow[t]{2}{*}{ Brain stem } & $45.02 \pm 1.15$ & $41.13 \pm 2.45$ & \\
\hline & $45.21(43.39-46.64)$ & $40.59(39.16-47.27)$ & $0.002 *$ \\
\hline \multirow{3}{*}{ Right eye } & & & \\
\hline & $25.23 \pm 11.16$ & $21.31 \pm 7.27$ & \\
\hline & $24.95(10.31-48.95)$ & $20.73(11.12-35.33)$ & 0.496 \\
\hline \multirow[t]{2}{*}{ Left eye } & $18.11 \pm 8.2$ & $29.26 \pm 8.05$ & \\
\hline & $14.58(9.14-35.72)$ & $29.3(11.62-42.73)$ & $0.019^{*}$ \\
\hline \multirow[t]{2}{*}{ Right lens } & $6.23 \pm 0.32$ & $6.5 \pm 0.43$ & \\
\hline & $6.15(5.83-6.7)$ & $6.61(5.55-7)$ & 0.054 \\
\hline \multirow[t]{2}{*}{ Left lens } & $6.06 \pm 0.86$ & $6.53 \pm 0.4$ & \\
\hline & $6.3(3.72-6.7)$ & $6.55(5.77-7.22)$ & 0.121 \\
\hline \multirow[t]{2}{*}{ Right optic nerve } & $35.92 \pm 12.34$ & $31.06 \pm 8.55$ & \\
\hline & $38.98(18.2-51.73)$ & $32.83(19.67-42.1)$ & 0.257 \\
\hline \multirow[t]{2}{*}{ Left optic nerve } & $28.58 \pm 10.67$ & $35.26 \pm 7.23$ & \\
\hline & $24.88(19.94-53.7)$ & $32.31(28.48-48.28)$ & $0.019^{*}$ \\
\hline \multirow[t]{2}{*}{ Optic chiasm } & $52.32 \pm 1.38$ & $52.77 \pm 0.74$ & \\
\hline & $52.56(50.61-54.14)$ & $52.69(51.75-53.76)$ & 0.496 \\
\hline Brain stem & $51.97 \pm 1.52$ & $51.36 \pm 2.55$ & \\
\hline & $52.32(49.3-54.3)$ & $52.02(46.19-53.78)$ & 1.000 \\
\hline
\end{tabular}

${ }^{*} \mathrm{P}<0.05$, compared with Mann Whitney U test, VMAT: Volumetric Modulated Arc Therapy, IMRT: Intensity-Modulated Radiotherapy, PTV: Planning target volume, $D_{95 \%}:$ The dose to cover $95 \%$ of organ at risk, $D_{\text {mean }}$ : Mean dose and $D_{\text {max }}$ : Maximum dose to cover the organ at risk 
duce cochlear impairing or hearing loss. $[4,5,8,11] \mathrm{Be}$ cause of several factors, including relatively low total prescription dose and poor prognosis associated with radiotherapy in patients with multiple brain tumors, potential radiation-induced toxicity to the OARs has been overlooked and underestimated.[15] Thus, in this retrospective study, we compared two modalities of radiotherapy, VMAT and IMRT, in the patients with brain tumor concerning the sparing effect on cochlea, by comparing dosimetric parameters. VMAT is a technique of rotational radiotherapy that delivers a highly conformal radiation dose to the target by simultaneously modulating gantry rotation, dose rate, and multileaf collimator pattern in linear accelerator.[16] Although VMAT system was claimed to be an advantageous conformal technique over the IMRT system in reducing the total number of MU and subsequently the beam on time, which may improve patient tolerance of treatment and potentially reduce leakage radiation dose to the patients, [16] in the present study, a significantly higher MU was detected in VMAT plan when compared with IMRT plan. However, VMAT plans may be superior concerning decreasing the dose to the OARs, including the optic and auditory structures. [15] In our study, sparing the cochlea was comparable among VMAT and IMRT plans about decreasing the applied dose, but VMAT was considerably surpassing to spare the eye and optic nerve but not the optic chiasm and brain stem compared with IMRT technique.

In clinical practice, radiotherapy has been observed to result in ear pain and fluid collection in the middle ear, which may cause otitis media and hearing loss. Radiation-induced hearing loss is a serious complication of radiotherapy in patients with brain tumors, which significantly affects the overall quality of life. $[17,18]$ The frequency of radiation-induced damage to ear function was reported as high as $37 \%$ in patients treated with IMRT.[11] By reducing the dose to the hearing apparatus, the incidence of hearing loss is likely to decline, and probably this is valid for both VMAT and IMRT planning since sparing the cochlea did not change the resultant doses to cover $95 \%$ of both total and contralateral cochlea. No difference was found in the mean, maximum and minimum doses to these structures for both techniques. In the future, a follow-up study is suggested to prove this consideration by calculating the ratio of hearing loss in a large population of patients with brain tumors

There are few publications focused on the protection of the hearing apparatus to compare between VMAT and IMRT. In a study by Vanetti et al., the ra- diation doses in VMAT and IMRT were compared for head and neck cancer, and VMAT plans outperformed IMRT plans concerning homogeneity and conformity in PTV, as well as providing a better sparing effect on the OARs.[19] In another study, Gao et al. compared the impacts of using SmartArc-based VMAT (VMAT-S) and step-and-shoot IMRT on hearing apparatus doses, as well as other involved OARs such as brain stem and spinal cord in the patient with nasopharyngeal carcinoma. They found that VMAT-S was significantly superior about PTV coverage and the protection of ear function compared with IMRT. The doses of middle ears, cochleas and vestibules were significantly lower in the VMATS plan.[11] However, in our study, the conformity and homogeneity indexes of VMAT and IMRT were the same, and the sparing effect for cochlea was also similar between two techniques. Moreover, IMRT was significantly superior over VMAT concerningPTV coverage at $46 \mathrm{~Gy}$. However, the doses of optical organs such as eye and optic nerve were significantly lower in the VMAT plan compared with the IMRT plan. These variances in results are inevitable since the sites tumors of the study population and the relevant exposed sites to the radiotherapies are varied among studies. To the best of our knowledge, this is the first report to compare VMAT and IMRT techniques in patients with brain tumors in terms of cochlear sparing.

Reducing the dose of radiotherapy and minimizing both acute and late toxicity of treatment in normal tissues is highly crucial for the patients and clinicians. [20] IMRT has become a modality of choice, allowing similar or better target coverage by providing more freedom in the beam arrangement and by achieving good conformity and sparing and avoiding normal tissues as compared to other conformal radiotherapies.[21] On the other hand, VMAT delivers a modulated beam in one or more arcs, mostly providing similar target coverage and normal tissue sparing as IMRT while substantially decreasing the treatment time. Despite, including limited numbers of patients, dosimetric analysis and radiotherapy planning studies have revealed the noninferiority of VMAT over IMRT in cancer patients.[21-23] To demonstrate the differences in planning quality between VMAT and IMRT methods for glioblastoma, Briere et al. analyzed the dose distributions of 90 clinical treatment plans, 45 patients treated with VMAT and 45 with IMRT. VMAT was found to be superior in sparing of the brainstem, the ipsilateral and contralateral cochlear and contralateral lens. They concluded that VMAT for glioblastoma patients could provide similar tar- 
get coverage, superior sparing of the brainstem and cochleae, and be delivered in a shorter period of time compared with IMRT.[24] As a limitation in our study, the treatment time was not analyzed, but we could not show any distinction among the patients with a brain tumor treated by VMAT or IMRT in terms of sparing the cochlea. However, our results of comparing the dosimetric variables between the two techniques are valuable to offer no inferiority of VMAT in comparison to IMRT. Hence, VMAT may suggest a clinical efficiency and the quality of the treatment experience by shortening the time of therapies in patients with intracranial tumors. Thus, the choice of two modalities for the treatment of cancers appears to base on the institutional or clinical experience, and suggested varied advantages and efficiencies among the types of cancers. The clinical outcomes of different planning in radiotherapies need further investigation.

\section{Conclusion}

Since VMAT and IMRT planning have the same effect on sparing the cochlea; no superiority can be suggested among these two plans during radiotherapy. The choice of plan for the treatment of brain tumors appears to base on the clinical experience, especially for patients who may experience hearing loss.

Peer-review: Externally peer-reviewed.

Conflict of Interest: None of the authors have any conflicts of interest or financial ties to disclose.

Ethics Committee Approval: The present study was approved by the Ethics Committee Approval (Number: 2017/28) for VMAT/IMRT planning and conducted in accordance with the principles of the WMA Declaration of Helsinki. Patient consent was not obligatory, as stated by the Ethics Committee of our institute.

Financial Support: None declared.

Authorship contributions: Concept - G.P.S.G., E.E.Ö.; Design - G.P.S.G., E.E.Ö.; Supervision - G.P.S.G., E.E.Ö.; Funding - E.E.Ö.; Materials - E.E.; Data collection and/or processing - G.P.S.G., E.E.Ö.; Data analysis and/or interpretation - G.P.S.G., E.E.Ö.; Literature search - G.P.S.G., E.E.Ö.; Writing - G.P.S.G., E.E.Ö.; Critical review - G.P.S.G., E.E.Ö.

\section{References}

1. Khuntia D, Brown P, Li J, Mehta MP. Whole-brain radiotherapy in the management of brain metastasis. J Clin Oncol 2006;24(8):1295-304.
2. Diaz AZ, Choi M. Radiation-associated toxicities in the treatment of high-grade gliomas. Semin Oncol 2014;41(4):532-40.

3. Bhandare N, Antonelli PJ, Morris CG, Malayapa RS, Mendenhall WM. Ototoxicity after radiotherapy for head and neck tumors. Int J Radiat Oncol Biol Phys 2007;67(2):469-79.

4. Hua C, Bass JK, Khan R, Kun LE, Merchant TE. Hearing loss after radiotherapy for pediatric brain tumors: effect of cochlear dose. Int J Radiat Oncol Biol Phys 2008;72(3):892-9.

5. Pan CC, Eisbruch A, Lee JS, Snorrason RM, Ten Haken RK, Kileny PR. Prospective study of inner ear radiation dose and hearing loss in head-and-neck cancer patients. Int J Radiat Oncol Biol Phys 2005;61(5):1393-402.

6. Cha J, Suh CO, Park K, Chang JH, Lee KS, Kim SH, et al. Feasibility and outcomes of hypofractionated simultaneous integrated boost-intensity modulated radiotherapy for malignant gliomas: a preliminary report. Yonsei Med J 2014;55(1):70-7.

7. Nakamatsu K, Suzuki M, Nishimura Y, Kanamori S, Koike R, Shibata T, et al. Treatment outcomes and dose-volume histogram analysis of simultaneous integrated boost method for malignant gliomas using intensity-modulated radiotherapy. Int J Clin Oncol 2008;13(1):48-53.

8. Woods K, Lee P, Kaprealian T, Yang I, Sheng K. Cochlea-sparing acoustic neuroma treatment with $4 \pi$ radiation therapy. Adv Radiat Oncol 2018;3(2):100-7.

9. Veldeman L, Madani I, Hulstaert F, De Meerleer G, Mareel M, De Neve W. Evidence behind use of intensity-modulated radiotherapy: a systematic review of comparative clinical studies. Lancet Oncol 2008;9(4):367-75.

10. Teoh M, Clark CH, Wood K, Whitaker S, Nisbet A. Volumetric modulated arc therapy: a review of current literature and clinical use in practice. Br J Radiol 2011;84(1007):967-96.

11. Gao J, Qian TL, Tao CZ, Zhang YH, Zhou Y, Yang J, et al. SmartArc-based volumetric modulated arc therapy can improve the middle ear, vestibule and cochlea sparing for locoregionally advanced nasopharyngeal carcinoma: a dosimetric comparison with step-andshoot intensity-modulated radiotherapy. Br J Radiol 2015;88(1053):20150052.

12. Fippel M. Fast Monte Carlo dose calculation for photon beams based on the VMC electron algorithm. Med Phys 1999;26(8):1466-75.

13. Mehta MP, Tsao MN, Whelan TJ, Morris DE, Hayman JA, Flickinger JC, et al. The American Society for Therapeutic Radiology and Oncology (ASTRO) evidence-based review of the role of radiosurgery for brain metastases. Int J Radiat Oncol Biol Phys 2005;63(1):37-46. 
14. Buglione M, Bandera L, Grisanti S, Pasinetti N, Borghetti P, Barbera F, et al. The impact of tumour histology and recursive partitioning analysis classification on the prognosis of patients treated with whole-brain hypofractionated radiotherapy for brain metastases: analysis of 382 patients. [Article in English, Italian] Radiol Med 2012;117(1):133-47.

15. Sood S, Pokhrel D, McClinton C, Lominska C, Badkul $\mathrm{R}$, Jiang $\mathrm{H}$, et al. Volumetric-modulated arc therapy (VMAT) for whole brain radiotherapy: not only for hippocampal sparing, but also for reduction of dose to organs at risk. Med Dosim 2017;42(4):375-83.

16. Rana S, Pokharel S, Zheng Y, Zhao L, Risalvato D, Vargas $\mathrm{C}$, et al. Treatment planning study comparing proton therapy, RapidArc and IMRT for a synchronous bilateral lung cancer case. Int J Cancer Ther Oncol 2014;2(2):020216.

17. Anteunis LJ, Wanders SL, Hendriks JJ, Langendijk JA, Manni JJ, de Jong JM. A prospective longitudinal study on radiation-induced hearing loss. Am J Surg 1994;168(5):408-11.

18. Petsuksiri J, Sermsree A, Thephamongkhol K, Keskool P, Thongyai K, Chansilpa Y, et al. Sensorineural hearing loss after concurrent chemoradiotherapy in nasopharyngeal cancer patients. Radiat Oncol 2011;6:19.

19. Vanetti E, Clivio A, Nicolini G, Fogliata A, GhoshLaskar S, Agarwal JP, et al. Volumetric modulated arc radiotherapy for carcinomas of the oro-phar- ynx, hypo-pharynx and larynx: a treatment planning comparison with fixed field IMRT. Radiother Oncol 2009;92(1):111-7.

20. Gao M, Li Q, Ning Z, Gu W, Huang J, Mu J, et al. Dosimetric comparison between step-shoot intensitymodulated radiotherapy and volumetric-modulated arc therapy for upper thoracic and cervical esophageal carcinoma. Med Dosim 2016;41(2):131-5.

21. Amelio D, Lorentini S, Schwarz M, Amichetti M. Intensity-modulated radiation therapy in newly diagnosed glioblastoma: a systematic review on clinical and technical issues. Radiother Oncol 2010;97(3):361-9.

22. Shaffer R, Nichol AM, Vollans E, Fong M, Nakano $\mathrm{S}$, Moiseenko V, et al. A comparison of volumetric modulated arc therapy and conventional intensity-modulated radiotherapy for frontal and temporal high-grade gliomas. Int J Radiat Oncol Biol Phys 2010;76(4):1177-84.

23. Panet-Raymond V, Ansbacher W, Zavgorodni S, Bendorffe B, Nichol A, Truong PT, et al. Coplanar versus noncoplanar intensity-modulated radiation therapy (IMRT) and volumetric-modulated arc therapy (VMAT) treatment planning for fronto-temporal highgrade glioma. J Appl Clin Med Phys 2012;13(4):3826.

24. Briere TM, McAleer MF, Levy LB, Yang JN. Sparing of normal tissues with volumetric arc radiation therapy for glioblastoma: single institution clinical experience. Radiat Oncol 2017;12(1):79. 